\title{
Barrier-controlled carrier transport in microcrystalline semiconducting materials: Description within a unified model
}

\author{
T. Weis ${ }^{a}$, R. Lipperheide ${ }^{a}$, U. Wille ${ }^{a, *}$, and S. Brehme ${ }^{b}$ \\ a Abteilung Theoretische Physik, Hahn-Meitner-Institut Berlin, Glienicker Str. 100, \\ D-14109 Berlin, Germany \\ b Abteilung Silizium-Photovoltaik, Hahn-Meitner-Institut Berlin, Kekuléstr. 5, \\ D-12489 Berlin, Germany
}

(November 19, 2018)

\begin{abstract}
A recently developed model that unifies the ballistic and diffusive transport mechanisms is applied in a theoretical study of carrier transport across potential barriers at grain boundaries in microcrystalline semiconducting materials. In the unified model, the conductance depends on the detailed structure of the band edge profile and in a nonlinear way on the carrier mean free path. Equilibrium band edge profiles are calculated within the trapping model for samples made up of a linear chain of identical grains. Quantum corrections allowing for tunneling are included in the calculation of electron mobilities. The dependence of the mobilities on carrier mean free path, grain length, number of grains, and temperature is examined, and appreciable departures from the results of the thermionic-field-emission model are found. Specifically, the unified model is applied in an analysis of Hall mobility data for n-type $\mu \mathrm{c}-\mathrm{Si}$ thin films in the range of thermally activated transport. Owing mainly to the effect of tunneling, potential barrier heights derived from the data are substantially larger than the activation energies of the Hall mobilities. The specific features of the unified model, however, cannot be resolved within the rather large uncertainties of the analysis.
\end{abstract}

PACS Numbers: 72.20.-i, 73.50.-h, 73.50.Dn, 73.50.Jt

*Author to whom correspondence should be addressed; electronic mail: wille@hmi.de 


\section{INTRODUCTION}

Over the past decades, a great deal of interest has been devoted to the study of the transport properties of microcrystalline semiconducting materials [1-5]. The commonly adopted viewpoint is that these properties are largely determined by potential barriers built up at grain boundaries due to charge carrier trapping into interface states [6]. Carrier transport across these barriers is mostly described in terms of the traditional thermionicemission model [7], or the thermionic-field-emission model [8,9] in which corrections allowing for quantum tunneling through the barriers are included.

Systematic studies of the transport properties in microcrystalline materials are usually based on measurements of Hall mobilities in thin films [1, 14. Seto [10] has been the first to perform a detailed theoretical analysis of Hall data, applying the grain-boundary trapping model in conjunction with the thermionic-emission model. For p-type $\mu \mathrm{c}-\mathrm{Si}$ thin films, potential barrier heights and trapping-state densities were deduced for different doping concentrations. In a large number of subsequent studies (see, e.g., Refs. [11 20], and references cited therein), Hall mobilities were analyzed with regard to their dependence on temperature, doping concentration, and film thickness. While, in general, these analyses have supported the picture of thermally activated transport over grain boundary barriers, the quantitative understanding of barrier-controlled transport appears to be incomplete yet. For example, the effect of tunneling [12] and the detailed form of the density of grain-boundary trapping states [21,22] need further clarification.

In the present paper, we concentrate on one particular aspect of the decription of barriercontrolled transport that does not seem to have been systematically studied until now, viz., the role played by the transport mechanism. In many practical cases, the magnitude of the carrier mean free path in microcrystalline material is comparable to the grain length, and so it appears indicated to consider departures of the transport mechanism from the commonly assumed thermionic-emission mechanism. A framework well suited to deal with this situation is provided by the unified transport model developed recently by the present authors [23]. This model unifies the ballistic and diffusive transport mechanisms in a generalization of the Drude model. The unified model is valid for arbitrary shape of the band edge profiles and for arbitrary magnitude of the carrier mean free path.

In the next section, the main features of the unified transport model are briefly summarized and discussed. In Sec. III, electron mobilities calculated within the unified model are presented and analyzed with regard to their dependence on various parameters. Comparison is made with the thermionic-field-emission model. Section IV deals with the application of the unified model in an analysis of Hall mobilities for n-type $\mu \mathrm{c}-\mathrm{Si}$ thin films. By suitably choosing the model parameters, grain-boundary potential barrier heights are inferred from the Hall data. Finally, in Sec. V, the contents of the paper are summarized and some concluding remarks are made.

\section{UNIFIED TRANSPORT MODEL}

In this section, we summarize and discuss the essential features of the unified transport

model [23]. A one-dimensional formulation is used in conjunction with the semiclassical 
approach. We restrict ourselves to considering the case of electrons (holes are described analogously).

The unified model is based on the idea that electrons move ballistically in the electric field over intervals with average length equal to a universal mean free path $l$. At the end of a ballistic interval, the electrons are thermalized into a state of local equilibrium characterized by a quasi-Fermi level $E_{\mathrm{F}}(x)$. The length $S$ of the sample is made up of random configurations of ballistic intervals. By appropriately averaging over these configurations, one arrives at a unified description of electron transport, which is valid for arbitrary magnitude of $l$ and for arbitrary shape of the conduction band edge profile $E_{\mathrm{c}}(x)$. The purely ballistic and purely diffusive transport mechanisms appear as limiting cases of this description.

For the present purpose, the principal result of the unified model is the formula for the zero-bias conductance per unit area,

$$
g=\frac{4 \pi e^{2} m^{*}}{\beta h^{3}} \ln \left(1+e^{-\beta E_{\mathrm{p}}}\right) \Gamma \frac{l}{L}
$$

$\left(\beta=1 / k_{\mathrm{B}} T\right)$, which allows for degeneracy and quantum tunneling. This formula comprises Eqs. (46) and (48) of Ref. [23], in which degeneracy and tunneling corrections, respectively, were taken into account separately. In Eq. (1) $E_{\mathrm{p}}=E_{\mathrm{c}}^{\mathrm{m}}-E_{\mathrm{F}}$, where $E_{\mathrm{c}}^{\mathrm{m}}$ is the maximum of the band edge profile $E_{\mathrm{c}}(x)$ for $0 \leq x \leq S$ and $E_{\mathrm{F}}$ is the Fermi level for zero bias (note that in the degenerate case, $E_{\mathrm{p}}$ may be negative). The quantity $\Gamma$ is the tunneling correction, and $L$ is the "effective transport length". In accordance with our description of carrier transport along classical trajectories, we treat tunneling in WKB approximation.

As we deal here with chains of identical grains, it is sufficient to consider tunneling through a single, symmetric potential barrier with maximum $E_{\mathrm{c}}^{\mathrm{m}}$ at $x=X$ (cf. Fig. 1). The correction $\Gamma$, defined as the ratio of the energy-integrated WKB and classical barrier transmission probabilities, is then given by

$$
\Gamma=1+\left[\ln \left(1+e^{-\beta E_{\mathrm{p}}}\right)\right]^{-1} \beta \int_{E_{\mathrm{c}}^{0}}^{E_{\mathrm{c}}^{\mathrm{m}}} d E \frac{T^{\mathrm{WKB}}(E)}{1+e^{\beta\left[E-E_{\mathrm{F}}\right]}},
$$

where $E_{\mathrm{c}}^{0}=E_{\mathrm{c}}(X-s / 2)$ is the value of the band edge profile at the center of the grain. For the WKB tunneling probability $T^{\mathrm{WKB}}(E)$, we have

$$
T^{\mathrm{WKB}}(E)=\exp \left(-(2 / \hbar) \int_{x_{1}}^{x_{2}} d x\left\{2 m^{*}\left[E_{\mathrm{c}}(x)-E\right]\right\}^{1 / 2}\right) .
$$

Here, $x_{1}$ and $x_{2}$ are the positions of the left and right turning point, respectively, of the classical electron motion at total energy $E$.

The effective transport length $L$ has the form

$$
L=l+\widetilde{S}+\widetilde{\Lambda}
$$

It comprises, aside from the mean free path $l$ which represents the ballistic contribution to electron transport, the "reduced sample length" $\widetilde{S}$ and the "shape term" $\widetilde{\Lambda}$. While $\widetilde{S}$ reflects the diffusive contribution, $\widetilde{\Lambda}$ represents the interplay between ballistic and diffusive transport. For a chain of $\nu$ identical grains, each of length $s$, the reduced sample length is given by 


$$
\widetilde{S}=\nu \widetilde{s}=\nu \Gamma \int_{X-s}^{X} d x F(x)
$$

where

$$
F(x)=\frac{\ln \left(1+e^{\beta\left[E_{\mathrm{F}}-E_{\mathrm{c}}^{\mathrm{m}}\right]}\right)}{\ln \left(1+e^{\beta\left[E_{\mathrm{F}}-E_{\mathrm{c}}(x)\right]}\right)} .
$$

For the shape term, we have

$$
\begin{aligned}
\widetilde{\Lambda}=(\nu-1) \tilde{\lambda} & =(\nu-1) \int_{X-s}^{X} d x e^{-2|x-(X-s / 2)| / l}[1-\Gamma F(x)] \\
& =(\nu-1)\left\{\left(1-e^{-s / l}\right) l-\Gamma \int_{X-s}^{X} d x e^{-2|x-(X-s / 2)| / l} F(x)\right\} .
\end{aligned}
$$

It is seen from Eq. (11) that, except for an overall factor, the quantity $R=\Gamma^{-1}(L / l)$ is the resistance of the chain. We split up $R$ into a net ballistic contribution $R_{\mathrm{b}}$ and a net diffusive contribution $R_{\mathrm{d}}$,

$$
R=\Gamma^{-1} \frac{L}{l}=R_{\mathrm{b}}+R_{\mathrm{d}} .
$$

Using Eqs. (14), (5), and (7), we find for the ballistic contribution (which is proportional to the inverse of the probability for transmission through the barriers)

$$
R_{\mathrm{b}}=\Gamma^{-1}\left[1+(\nu-1)\left(1-e^{-s / l}\right)\right] .
$$

In this expression, one barrier contributes fully ballistically, while the contribution of the other $\nu-1$ barriers is weighted by the probability $1-\exp (-s / l)$ that one or more collisions (thermalizations) occur within the distance $s$ in the valley between one barrier and the next. Without such collisions, the barrier at the end of the valley would not contribute; it would be "eclipsed" by the preceding barrier (cf. Ref. [23]). The contribution of each barrier contains the factor $\Gamma^{-1}$, which takes into account the reduction of the resistance arising from tunneling. For the diffusive contribution, we have

$$
R_{\mathrm{d}}=\int_{X-s}^{X} \frac{d x}{l}\left[\nu-(\nu-1) e^{-2|x-(X-s / 2)| / l}\right] F(x) .
$$

Here, each of the $\nu$ intervals contributes its diffusive resistance, but this is diminished by the ballistic contributions of the $\nu-1$ valleys, which enter in the weight factors $\exp [-2 \mid x-$ $(X-s / 2) \mid / l]$ representing the probabilities for collision-free motion.

The energy $E$ above which the tunneling probability $T^{\mathrm{WKB}}(E)$ becomes appreciable is usually larger than $E_{\mathrm{c}}^{0}$. The associated turning points lie within a region of width $\Delta$, which defines the "tunneling interval" 23], i.e., the distance across which tunneling can occur (cf. Fig. 1). Since tunneling is a ballistic process, one must have $\Delta \ll l$, and we must exclude ballistic interval lengths smaller than $\Delta$ when averaging over the configurations made up of these intervals 23]. This should entail a relative reduction of order $\Delta / l$ of all integrals over $x$ in Eqs. (5), (7), and (10). In the application considered in Sec. IV, we have $\Delta / l<0.2$, and in view of the other simplifications of the model we omit the corresponding correction in our calculations. This procedure may be viewed as a heuristic description of the simultaneous occurrence of tunneling and diffusion. It differs from that of Ref. [23], where the tunneling interval has been excluded from the above-mentioned integrals. 


\section{ELECTRON MOBILITIES}

In the unified transport model, the (effective) electron mobility $\mu$ for a chain of $\nu$ identical grains of length $s$ is given by

$$
\mu=g \frac{\nu s}{e \bar{n}}=\frac{4 \pi e m^{*}}{\beta h^{3}} \frac{\nu s}{\bar{n}} \ln \left(1+e^{-\beta E_{\mathrm{p}}}\right) \Gamma \frac{l}{L},
$$

where $g$ is the conductance of Eq. (1) and $\bar{n}$ is the electron concentration averaged over a grain. The effective length $L$ depends explicitly on the mean free path $l$ and on the number of grains, $\nu$ [cf. Eqs. (4)-(7)]. In particular,

$$
\mu \propto \begin{cases}l /(l+\widetilde{s}) & \text { for } \nu=1 \\ l /(\widetilde{\lambda}+\widetilde{s}) & \text { for } \nu \rightarrow \infty .\end{cases}
$$

Note that the expression (11) refers to an ideal, one-dimensional situation with strictly homogeneous grain boundaries.

Aside from the explicit dependence of the mobility $\mu$ on the mean free path $l$, an implicit dependence on $l$ arises from the dependence of the band edge profile $E_{\mathrm{c}}(x)$ on the donor concentration $N_{\mathrm{d}}$. Adopting a relation between $l$ and $N_{\mathrm{d}}$ obtained from Ref. [24], we examine here the characteristic behavior of $\mu$ as a function of mean free path $l$ (or donor concentration $N_{\mathrm{d}}$ ), grain number $\nu$, and grain length $s$, and compare it to the behavior found for thermionic field emission. For that mechanism, the mobility is obtained by setting $\nu=1$ and $L=l$ in Eq. (11) [the $l$-dependence of the mobility is then determined solely by the implicit $l$ dependence of the band edge profile].

Using Fermi-Dirac statistics and assuming the grain boundaries to be infinitesimally narrow, we calculate equilibrium band edge profiles $E_{\mathrm{c}}(x)$ within the trapping model by numerically solving the nonlinear Poisson equation (thereby going beyond the depletion approximation [10]) for a single grain under periodic boundary conditions [23, 25, 26]. The density $D_{\mathrm{t}}(E)$ of trapping states at a grain boundary is taken as the sum of a term corresponding to a deep level at energy $E_{0}$ and an exponential describing tail states [22] extending from the bulk conduction band edge at $E_{\mathrm{c}}$ down into the gap, i.e.,

$$
D_{\mathrm{t}}(E)=N_{0} \delta\left(E-E_{0}\right)+\kappa \mathrm{e}^{-\left(E_{\mathrm{c}}-E\right) / \epsilon} .
$$

Electron mobilities for n-type $\mu \mathrm{c}$-Si at room temperature $(T=300 \mathrm{~K})$, calculated from the unified-model expression (11), are displayed in Figs. 2 -5 as a function of the parameters $l, \nu$, and $s$. For the parameters in the trapping-state density $D_{\mathrm{t}}(E)$, we have used values that emerged from our previous analysis of Hall mobility data for phosphorus-doped $\mu \mathrm{c}-\mathrm{Si}$ within the thermionic-field-emission model including a fully quantal treatment of tunneling [27], viz., $N_{0}=\alpha_{0}+\alpha_{1} N_{\mathrm{d}}\left(\alpha_{0}=3.54 \times 10^{12} \mathrm{~cm}^{-2}, \alpha_{1}=1.03 \times 10^{-7} \mathrm{~cm}\right), E_{0}=\left(E_{\mathrm{v}}+E_{\mathrm{c}}\right) / 2$ $\left(E_{\mathrm{v}}=\right.$ bulk valence band edge $), \kappa=1 \times 10^{15} \mathrm{~cm}^{-2} \mathrm{eV}^{-1}, \epsilon=28 \mathrm{meV}$. For exhibiting characteristic features of the unified model, this choice appears adequate. We will reconsider the determination of the parameter values in the trapping-state density in Sec. IV where Hall mobility data are analyzed within the unified model.

In Fig. 2, mobilities are shown as a function of mean free path $l$ (or of donor concentration $\left.N_{\mathrm{d}}\right)$ for a single grain $(\nu=1)$ and for a chain of many grains $(\nu \rightarrow \infty)$ [cf. Eq. (12)]. 
For comparison, the mobility of the thermionic-field-emission model is also shown. The pronounced minimum observed in all curves is associated with the transition from complete depletion of the grains to partial depletion when the donor concentration increases beyond a critical value [10]. In the range $l \approx s$, the unified-model mobility for $\nu \rightarrow \infty$ is larger by about $50 \%$, on average, than that for $\nu=1$. For larger $l$, the ratio of these two mobilities tends to diverge, with a variation proportional to $l / s$ when $l \gg s$ [cf. Eqs. (7) and (12)].

The transport mechanism in the unified model becomes purely ballistic in the large-l limit. Therefore, for a single grain, the corresponding mobility shown in Fig. 2 approaches that for the thermionic-field-emission mechanism which is also purely ballistic. On the other hand, for a chain of grains, the thermionic-field-emission model (and, of course, the thermionic-emission model as well) identifies the conductivity, and hence also the mobility, of a single grain with that of the whole chain. It thus tacitly assumes that the mean free path $l$ is long compared to the width of the barriers but short compared to the length of the grains, so that while moving through the grains, the electrons are thermalized and "face" each grain boundary barrier with the same thermal distribution as when passing over the previous one (cf. Sec. 4.1 of Ref. [23]). The thermionic-emission model is then no longer a purely ballistic model; for $\nu>1$, its results for large $l$ must differ from those of the unified model, in which the barriers begin to "eclipse" each other when $l$ becomes larger than the distance $s$ between them.

The influence of tunneling on the unified-model mobilities is illustrated in Fig. 3. The tunneling correction $\Gamma$ given by Eq. (2) is seen to rise rapidly with decreasing mean free path $l$ in the range $l<30 \mathrm{~nm}$. This behavior is traced to the rapid narrowing of the grain-boundary barriers (and to the concomitant increase in their transparency) when the donor concentration $N_{\mathrm{d}}$ increases beyond $10^{18} \mathrm{~cm}^{-3}$. In Eq. (11), the quantity $\Gamma$ enters as an overall factor and via the $\Gamma$-dependence of the effective length $L$ in the denominator [cf. Eqs. (4), (5), and (7)]. It appears that the latter dependence has small influence only, and thus tunneling enhances the mobility essentially by a factor $\Gamma$ over its classical value.

In Fig. 1 , unified-model mobilities are shown as a function of the number of grains, $\nu$. Sizeable variations of the mobility are restricted to the range of small $\nu$ and very low donor concentration $N_{\mathrm{d}}$. The $\nu$-dependence of the mobility arises from the eclipsing effect, which is most notable when comparing the transport across one barrier $(\nu=1)$ with that across two or three barriers $(\nu=2,3)$. As the number of barriers increases, the eclipsing effect of the added barriers becomes negligible, and the mobility becomes independent of $\nu$ [cf. Eq. (12)] (but not equal to that for a single grain).

The dependence of the mobilities of the unified and the thermionic-field-emission models

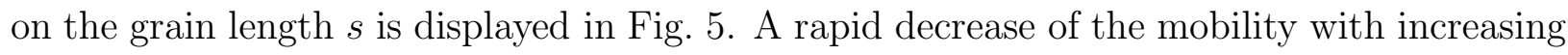
$s$ is observed at low donor concentration close to the minimum in the $N_{\mathrm{d}}$-dependence of $\mu$ appearing in Fig. 目 (cf. also Ref. [10]). For very low $N_{\mathrm{d}}$, there are strong discrepancies between the results of the unified model and those of the thermionic-field-emission model, in particular in the range of small $s$.

For large numbers of grains $(\nu \rightarrow \infty)$ and for mean free paths comparable to the grain length $(l \approx s)$, i.e., for parameter values encountered in many experimental studies, the results of Figs. 2 - 5 show that (i) the unified-model mobility is fairly close to the mobility of the thermionic-field-emission model; (ii) tunneling accounts for up to $50 \%$ of the unifiedmodel mobility; (iii) the unified-model mobility is virtually independent of the number of 
grains; (iv) as a function of grain length, the unified-model mobility exhibits rapid variations when the donor concentration is low.

\section{APPLICATION: ANALYSIS OF HALL MOBILITIES}

In this section, we apply the unified transport model in an analysis of Hall mobility data for thin films of n-type $\mu \mathrm{c}$-Si. Following common practice [1], we identify the effective mobility $\mu$ given by expression (11) with the Hall mobility $\mu_{\mathrm{H}}$. By fitting, in the range of thermally activated transport, the temperature dependence of $\mu$ to that of $\mu_{\mathrm{H}}$, we infer true potential barrier heights, which solely reflect structural properties of the grain boundaries. The deviations of the Hall activation energies from the true barrier heights exhibit the effect of the transport mechanism on the mobilities.

\section{A. Activation energies and barrier heights}

Our analysis is based on Hall data obtained for samples of highly phosphorus-doped $\mu$ c$\mathrm{Si}: H$ thin films, with thickness varying between 0.15 and $0.28 \mu \mathrm{m}$ and average grain length $s \approx 15 \mathrm{~nm}$ (for details, see Refs. [19,20]). In Fig. 6, the temperature dependence of the Hall mobilities $\mu_{\mathrm{H}}$ is shown in an Arrhenius plot. Fitting straight lines to the mobilities in the high- $T$ range, i.e., assuming the mobilities there to have the form

$$
\mu_{\mathrm{H}}=\mu_{\mathrm{H}}^{0} \exp \left(-E_{\mathrm{a}} / k_{\mathrm{B}} T\right)
$$

we have determined values for the preexponentials $\mu_{\mathrm{H}}^{0}$ and the activation energies $E_{\mathrm{a}}$ for the different samples (by restricting ourselves to the high- $T$ range, we avoid entering a discussion of the intricacies of curved Arrhenius behavior; cf. Ref. [28], and references cited therein). In Table I, the values of $\mu_{\mathrm{H}}^{0}$ and $E_{\mathrm{a}}$ are listed along with donor concentrations $N_{\mathrm{d}}$ estimated 119 from the electron concentrations by using Seto's model [10. The mean free paths $l$ corresponding to the $N_{\mathrm{d}}$-values vary between $10 \mathrm{~nm}$ and $20 \mathrm{~nm}$ (cf. inset in Fig. 2), i.e., the condition $l \approx s$ is fulfilled.

In Fig. 7, the temperature dependence of the mobilities of the unified model (for grain number $\nu \rightarrow \infty$ ) and the thermionic-field-emission model for grain length $s=15 \mathrm{~nm}$ and different values of the donor concentration $N_{\mathrm{d}}$ is displayed in an Arrhenius plot. For the parameters in the trapping-state density $D_{\mathrm{t}}(E)$, the same values as in the calculations shown in Figs. 25 were used. Major deviations from pure Arrhenius behavior are observed only for the unified-model results at $N_{\mathrm{d}}=3 \times 10^{20} \mathrm{~cm}^{-3}$ and low temperatures. These may be attributed to the strong temperature dependence of the Fermi level in that parameter range and its effect on the terms $\widetilde{\lambda}$ and $\widetilde{s}$ [cf. Eqs. (11) and (12)]. In those temperature ranges where the different data sets in Fig. 6 exhibit Arrhenius behavior, the temperature dependence of the corresponding theoretical mobilities can be well approximated, in the vicinity of a fixed temperature $T_{0}$, by an Arrhenius formula

$$
\mu=\mu_{0} \exp \left(-E_{\mathrm{b}}^{\mathrm{eff}} / k_{\mathrm{B}} T\right)
$$

where the preexponential $\mu_{0}$ and the "effective barrier height" $E_{\mathrm{b}}^{\text {eff }}$ depend weakly on $T_{0}$. We then can identify $E_{\mathrm{b}}^{\text {eff }}$, evaluated at a suitably chosen $T_{0}$-value, with the activation energy 
$E_{\mathrm{a}}$ derived from the experimental data. In the following, we use $T_{0}=300 \mathrm{~K}$ throughout; this value is roughly equal to the average of the centers of those $T$-intervals over which the Hall data of Fig. 6 exhibit Arrhenius behavior.

In order to infer true barrier heights, we match the effective barrier heights $E_{\mathrm{b}}^{\text {eff }}$ to the activation energies $E_{\mathrm{a}}$ given in Table I by adjusting the values of the parameters in the trapping-state density $D_{\mathrm{t}}(E)$. In our previous analysis of Hall mobilities within the thermionic-field-emission model [27], we have assigned fixed values to the parameters $E_{0}, \kappa$, and $\epsilon$, and subsequently adjusted the value of the area density $N_{0}$ independently for each sample, i.e., for each donor concentration $N_{\mathrm{d}}$, such as to obtain $E_{\mathrm{b}}^{\text {eff }}=E_{\mathrm{a}}$. The resulting $N_{0}$-values can be accurately fitted by means of the interpolation formula $N_{0}=\alpha_{0}+\alpha_{1} N_{\mathrm{d}}$ (cf. Sec. III).

Here, within the unified model, we adopt the matching procedure of Ref. [27], using, in particular, the same values for the parameters $E_{0}, \kappa$, and $\epsilon$ (cf. Sec. III). The adjusted values of the area density $N_{0}$ for the different donor concentrations $N_{\mathrm{d}}$ can again be interpolated by a linear expression, with parameter values $\alpha_{0}=3.79 \times 10^{12} \mathrm{~cm}^{-2}, \alpha_{1}=7.04 \times 10^{-8} \mathrm{~cm}$. While the value of $\alpha_{0}$ is close to that of Ref. [27], the value of $\alpha_{1}$ is smaller by about $30 \%$. Varying the values of $E_{0}, \kappa$, and $\epsilon$ within reasonable bounds does not lead to a substantial change in the adjusted values of $N_{0}$.

Using the interpolation formula for $N_{0}$ as a function of $N_{\mathrm{d}}$, we have calculated from Eqs. (11) and (15) a continuous curve for the $N_{\mathrm{d}}$-dependence of the effective barrier height $E_{\mathrm{b}}^{\text {eff }}$, which is displayed in Fig. 8 along with the activation energies $E_{\mathrm{a}}$. Also shown are the results of the thermionic-field-emission model, calculated with the same set of parameter values as used in the unified-model calculations. In obtaining the classical unified-model results for $E_{\mathrm{b}}^{\text {eff }}[\Gamma=1$ in Eq. (11)] included in Fig. 8, an independent adjustment of the parameter values in the trapping-state density $D_{\mathrm{t}}$ was performed. With $\kappa=5 \times 10^{14} \mathrm{~cm}^{-2} \mathrm{eV}^{-1}$ and $\epsilon=30 \mathrm{meV}$, a good fit to the activation energies was achieved by choosing for the area density $N_{0}$ the constant $\left(N_{\mathrm{d}}\right.$-independent $)$ value $2.89 \times 10^{12} \mathrm{~cm}^{-2}$.

The true grain-boundary barrier height $E_{\mathrm{b}}$, defined here as $E_{\mathrm{b}}=E_{\mathrm{c}}^{\mathrm{m}}-E_{\mathrm{c}}^{0}$ (i.e., identified with the "band bending" [1]), is directly read from the band edge profile $E_{\mathrm{c}}(x)$ calculated with the adjusted parameter values in the trapping-state density. Referring the barrier height to the profile at the center of the grain, $E_{\mathrm{c}}^{0}$, is appropriate when discussing results in which tunneling is taken into account [cf. Eq. (2)]. In Fig. 9, we show barrier heights $E_{\mathrm{b}}$ for the full and the classical unified model as a function of donor concentration $N_{\mathrm{d}}$. For direct comparison, we include the corresponding results for $E_{\mathrm{b}}^{\text {eff }}$ (cf. Fig. 8).

\section{B. Discussion}

The principal result of our analysis of Hall mobilities is that the true potential barrier heights derived within the unified model deviate substantially from the Hall activation energies. Considering the results presented in Figs. 8 and 9 in detail, we conclude that although the unified and thermionic-field-emission models generally yield appreciably different values for the electron mobilities (cf. Sec. III), the effective barrier heights obtained from the two models are in close agreement when one and the same (adjusted) set of parameters in the trapping-state density is used, thus implying identical values for the true barrier heights. In the present analysis, therefore, the effective barrier heights turn out to be not sufficiently 
sensitive to either type of transport mechanism; the subtle effects of the interplay between ballistic and diffusive transport, which are taken into account in the unified model, cannot be resolved within the uncertainties of the analysis. In the unified model, as in the thermionicfield-emission model, the transport mechanism manifests itself essentially through the effect of tunneling, which causes the true barrier heights to deviate appreciably from the effective heights, in particular for large donor concentrations.

It is seen that the true barrier heights derived within the classical approximation come closer to the effective heights, i.e., to the Hall activation energies. Finally, it is found that the adjusted values of the area density of the trapping states rise rapidly with increasing donor concentration when tunneling is taken into account. In the classical description, on the other hand, the area density exhibits a very weak dependence on donor concentration. The latter result is in line with the finding obtained by Seto [10] for p-type $\mu \mathrm{c}$-Si thin films.

In order to assess the general reliability of the present description and the relevance of the results of the analysis of Hall data, a number of remarks are in order.

(i) In our description of barrier-controlled transport, we have disregarded a number of effects whose consideration may quantitatively alter our results. We have confined ourselves to a one-dimensional formulation and have treated chains of identical grains only. Fluctuations in grain length, the non-planarity and inhomogeneity of the grain boundaries, and the perturbation caused by column boundaries have not been taken into account.

(ii) The absolute values of the electron mobilities calculated for n-type $\mu \mathrm{c}-\mathrm{Si}$ are by about one order of magnitude larger than the experimental values (cf. Figs. 6 and 7). Inhomogeneities in the grain-boundary properties that are not allowed for in our one-dimensional treatment are likely to be responsible [3, 16] for this feature.

(iii) There is a zone of disorder of finite extension at real grain boundaries (albeit estimated to be very narrow (width $<1 \mathrm{~nm}$ ) in $\mu \mathrm{c}-\mathrm{Si}[2]$ ). Thus, the band edge profiles calculated under the assumption of infinitesimally narrow grain boundaries will have no physical meaning in the close vicinity of the boundary. Accordingly, with increasing narrowing of the barriers, i.e., with increasing donor concentration, the uncertainty in the values inferred for the true barrier height $E_{\mathrm{b}}$ rises. For $N_{\mathrm{d}}>10^{20} \mathrm{~cm}^{-3}$, the calculated widths of the barriers are smaller than $1 \mathrm{~nm}$, so that in this range no significance can be attached to the values of $E_{\mathrm{b}}$ (cf. Fig. 9, dashed parts of upper curves).

(iv) In our analysis of Hall mobilities, uncertainties (aside from those associated with the basic limitations of our theoretical treatment) arise mainly from inaccuracies in the $N_{\mathrm{d}^{-}}$ values assigned to the different samples. The finite film thickness and its variation from sample to sample may also be a source of uncertainty.

\section{SUMMARY AND CONCLUSIONS}

In this paper, carrier transport across grain-boundary potential barriers in microcrystalline semiconducting materials has been theoretically studied with the aim to elucidate the role played by the transport mechanism. To this end, a recently developed model that unifies the ballistic and diffusive mechanisms was applied in the calculation of electron mobilities. A one-dimensional formulation of the model was used in conjunction with the trapping model. Quantum corrections allowing for tunneling were taken into account within the WKB approximation. 
In order to exhibit general features of the unified model, electron mobilities for $\mu \mathrm{c}-\mathrm{Si}$ have been calculated as a function of various parameters, such as carrier mean free path, grain length, number of grains, and temperature. Sizeable deviations from the results of the thermionic-field-emission model were found, particularly for large numbers of grains and for small grain lengths at low donor concentration.

Furthermore, we have confronted the unified transport model with experimental results by analyzing Hall mobilities for highly phosphorus-doped $\mu \mathrm{c}-\mathrm{Si}: \mathrm{H}$ thin films in the range of thermally activated transport. From the Hall activation energies, "true" grain-boundary barrier heights were determined by matching effective barrier heights deduced from the unified-model mobilities to the activation energies. The true heights turn out to be substantially larger than the activation energies, mainly owing to the effect of tunneling. The area density of trapping states obtained from the matching procedure rises rapidly with increasing donor concentration if tunneling is included in the unified-model calculation, but is virtually independent of donor concentration in the classical case.

In view of the limitations of our model description of carrier transport and of the uncertainties of our analysis of Hall mobilities, it would be premature to draw general conclusions from the results of the present study. Our results indicate, however, that in many cases of practical interest the thermionic-field-emission model may provide a sufficiently good description of barrier-controlled carrier transport. In order to reach definitive conclusions on the relevance of the unified transport model, more general versions of this model have to be worked out and systematically applied in the analysis of transport properties of microcrystalline semiconducting materials. 


\section{REFERENCES}

[1] J. W. Orton and M. J. Powell, Rep. Prog. Phys. 43, 1263 (1980).

[2] C. R. M. Grovenor, J. Phys. C: Solid State Phys. 18, 4079 (1985).

[3] T. Kamins, Polycrystalline Silicon for Integrated Circuit Applications (Kluwer, Boston, 1986), chap. 5.

[4] G. Willeke, in Amorphous and Microcrystalline Semiconductor Devices, Vol. II: Materials and Device Physics, edited by J. Kanicki (Artech House, London, 1991), p. 55.

[5] Proceedings of the Eighteenth International Conference on Amorphous and Microcrystalline Semiconductors, edited by S. Wagner, P. C. Taylor, R. A. Street, and E. A. Schiff (North-Holland, Amsterdam, 2000) [J. Non-Cryst. Solids 266-269 (2000)], and proceedings of previous conferences of this series.

[6] W. E. Taylor, N. H. Odell, and H. Y. Fan, Phys. Rev. 88, 867 (1952).

[7] H. A. Bethe, MIT Radiat. Lab. Rep. 43-12 (1942) [reprinted in Semiconductor Devices: Pioneering Papers, edited by S. M. Sze (World Scientific, Singapore, 1991), p. 387].

[8] W. W. Dolan and W. P. Dyke, Phys. Rev. 95, 327 (1954).

[9] R. Stratton, Phys. Rev. 125, 67 (1962).

[10] J. Y. W. Seto, J. Appl. Phys. 46, 5247 (1975).

[11] J. W. Orton, B. J. Goldsmith, M. J. Powell, and J. H. Chapman, Appl. Phys. Lett. 37, 557 (1980).

[12] A. P. Roth and D. F. Williams, J. Appl. Phys. 52, 6685 (1981).

[13] H. Richter and L. Ley, J. Appl. Phys. 52, 7281 (1981).

[14] M. Nakata and I. Shimizu, Mater. Res. Soc. Symp. Proc. 283, 591 (1993).

[15] D. He, N. Okada, C. M. Fortmann, and I. Shimizu, J. Appl. Phys. 76, 4728 (1994).

[16] M. W. J. Prins, K.-O. Grosse-Holz, J. F. M. Cillessen, and L. F. Feiner, J. Appl. Phys. 83, 888 (1998).

[17] K. Nakahata, A. Miida, T. Kamiya, C. M. Fortmann, and I. Shimizu, Thin Solid Films 337, 45 (1999).

[18] R. Carius, J. Müller, F. Finger, N. Harder, and P. Hapke, in Thin Film Materials and Devices - Developments in Science and Technology, edited by J. M. Marshall, N. Kirov, A. Vavrek, and J. M. Maud (World Scientific, Singapore, 1999), p. 157.

[19] S. Brehme, P. Kanschat, and W. Fuhs, Mat. Res. Soc. Symp. Proc. 609, A32.2 (2000).

[20] S. Brehme, P. Kanschat, T. Weis, K. Lips, and W. Fuhs, Solid State Phenomena 80-81, 225 (2001).

[21] G. E. Pike and C. H. Seager, J. Appl. Phys. 50, 3414 (1979).

[22] J. H. Werner, in Proceedings of the Sixth International Symposium on the Structure and Properties of Dislocations in Semiconductors, Oxford, 1989, edited by S. G. Roberts, D. B. Holt, and P. R. Wilshaw (IOP Publishing, Bristol, 1989) [Inst. Phys. Conf. Ser. No. 104], p. 63 (1989).

[23] R. Lipperheide, T. Weis, and U. Wille, J. Phys.: Condens. Matter 13, 3347 (2001).

[24] N. D. Arora, J. R. Hauser, and D. J. Roulston, IEEE Trans. Electron Devices ED-29, $292(1982)$.

[25] T. Weis, Ph. D. Thesis, Free University Berlin (1999).

[26] R. Lipperheide, T. Weis, and U. Wille, Sol. Energy Mater. Sol. Cells 65, 157 (2001).

[27] T. Weis, S. Brehme, P. Kanschat, W. Fuhs, R. Lipperheide, and U. Wille, J. Non-Cryst. Solids 299-302, 380 (2002). 
[28] J. H. Werner, Solid State Phenomena 37-38, 213 (1994). 


\section{TABLES}

TABLE I. Preexponentials $\mu_{\mathrm{H}}^{0}$ and activation energies $E_{\mathrm{a}}$ for different samples of phosphorusdoped $\mu \mathrm{c}-\mathrm{Si}: \mathrm{H}$ thin films with donor concentrations $N_{\mathrm{d}}$, obtained by fitting expression (14) to the Hall mobility data shown in Fig. 6 .

\begin{tabular}{|c||c|c|c|}
\hline$\#$ & $N_{\mathrm{d}}\left(10^{18} \mathrm{~cm}^{-3}\right)$ & $\mu_{\mathrm{H}}^{0}\left(\mathrm{~cm}^{2} \mathrm{~V}^{-1} \mathrm{~s}^{-1}\right)$ & $E_{\mathrm{a}}(\mathrm{meV})$ \\
\hline 1 & 1.7 & 5.6 & 55 \\
2 & 2.8 & 5.6 & 47 \\
3 & 9.3 & 5.5 & 31 \\
4 & 30 & 7.7 & 25 \\
5 & 72 & 5.2 & 18 \\
6 & 125 & 3.7 & 17 \\
7 & 292 & 2.6 & 11 \\
\hline
\end{tabular}




\section{FIGURES}

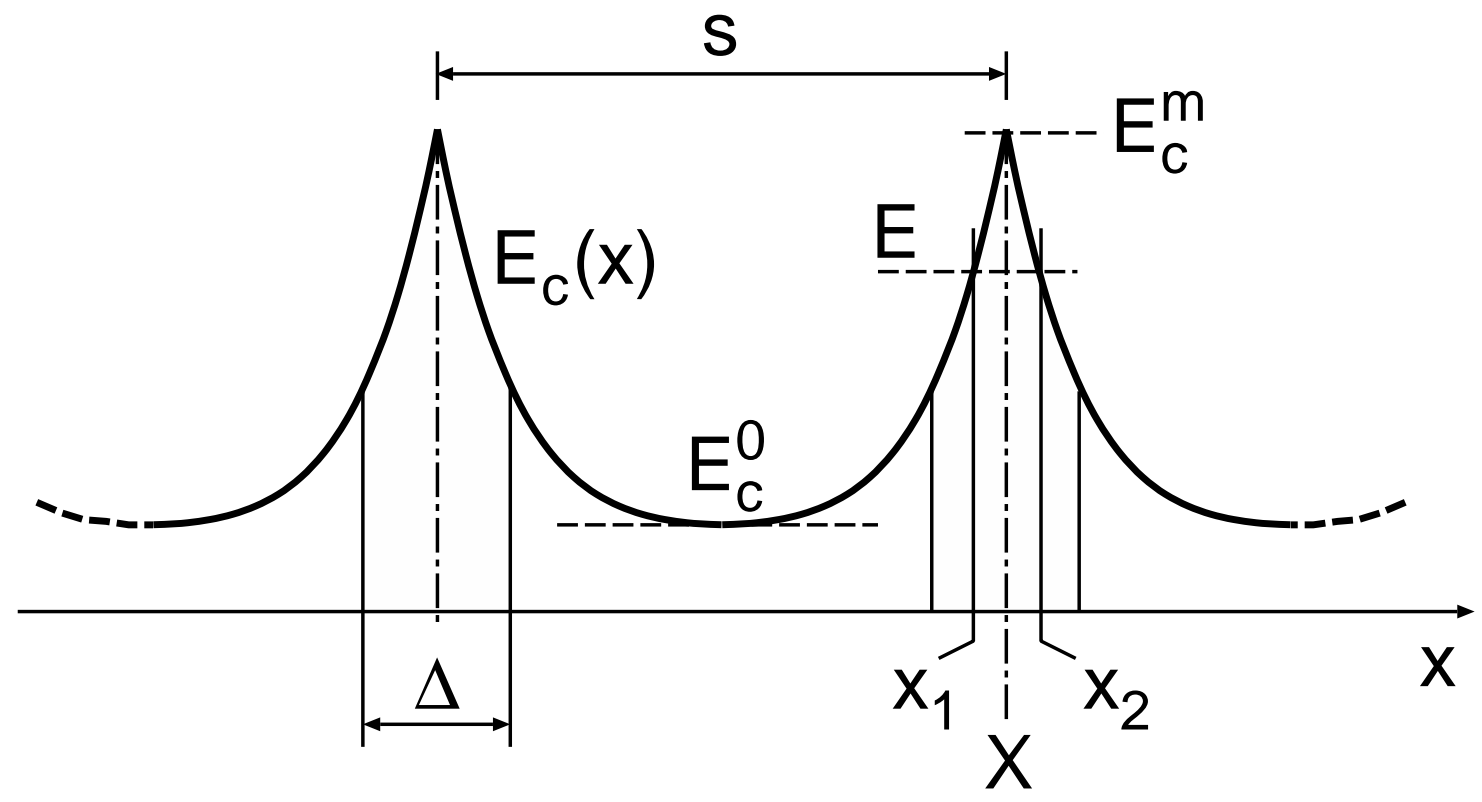

FIG. 1. Schematic equilibrium band edge profile for a chain of identical grains. 


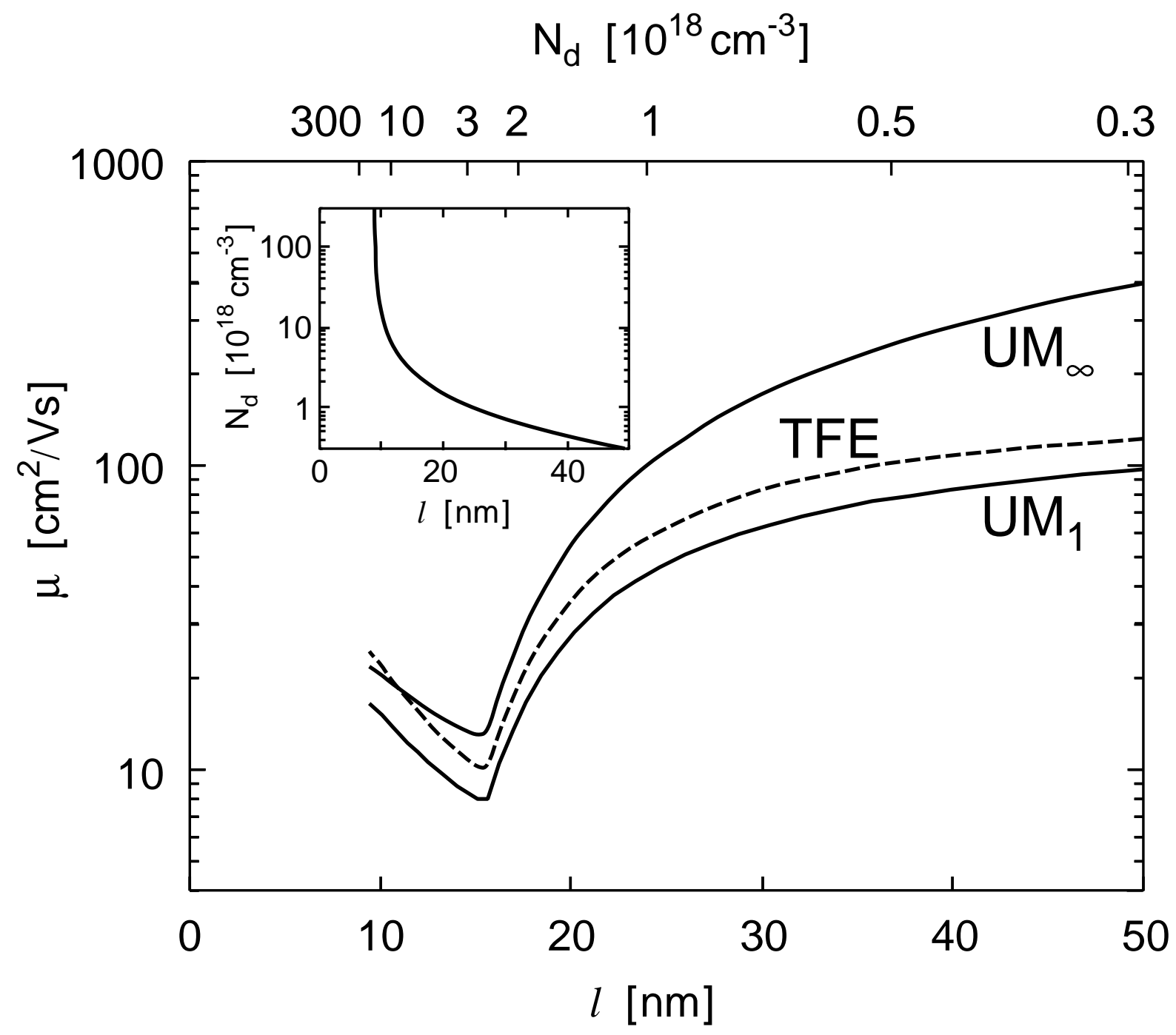

FIG. 2. Theoretical electron mobilities for n-type $\mu \mathrm{c}-\mathrm{Si}$ with grain length $s=15 \mathrm{~nm}$ at temperature $T=300 \mathrm{~K}$, plotted as a function of mean free path $l$ (lower abscissa) [or of donor concentration $N_{\mathrm{d}}$ (upper abscissa)]. In the conversion of $N_{\mathrm{d}}$ into $l$ (cf. inset), a relation given in Ref. [24] was used. Solid curves: mobilities calculated from the unified-model expression (11); curve $\mathrm{UM}_{1}$ : single grain, curve $\mathrm{UM}_{\infty}$ : chain of many grains. Dashed curve: mobility in the thermionic-field-emission (TFE) model. For parameter values in the trapping-state density (13), see text. 


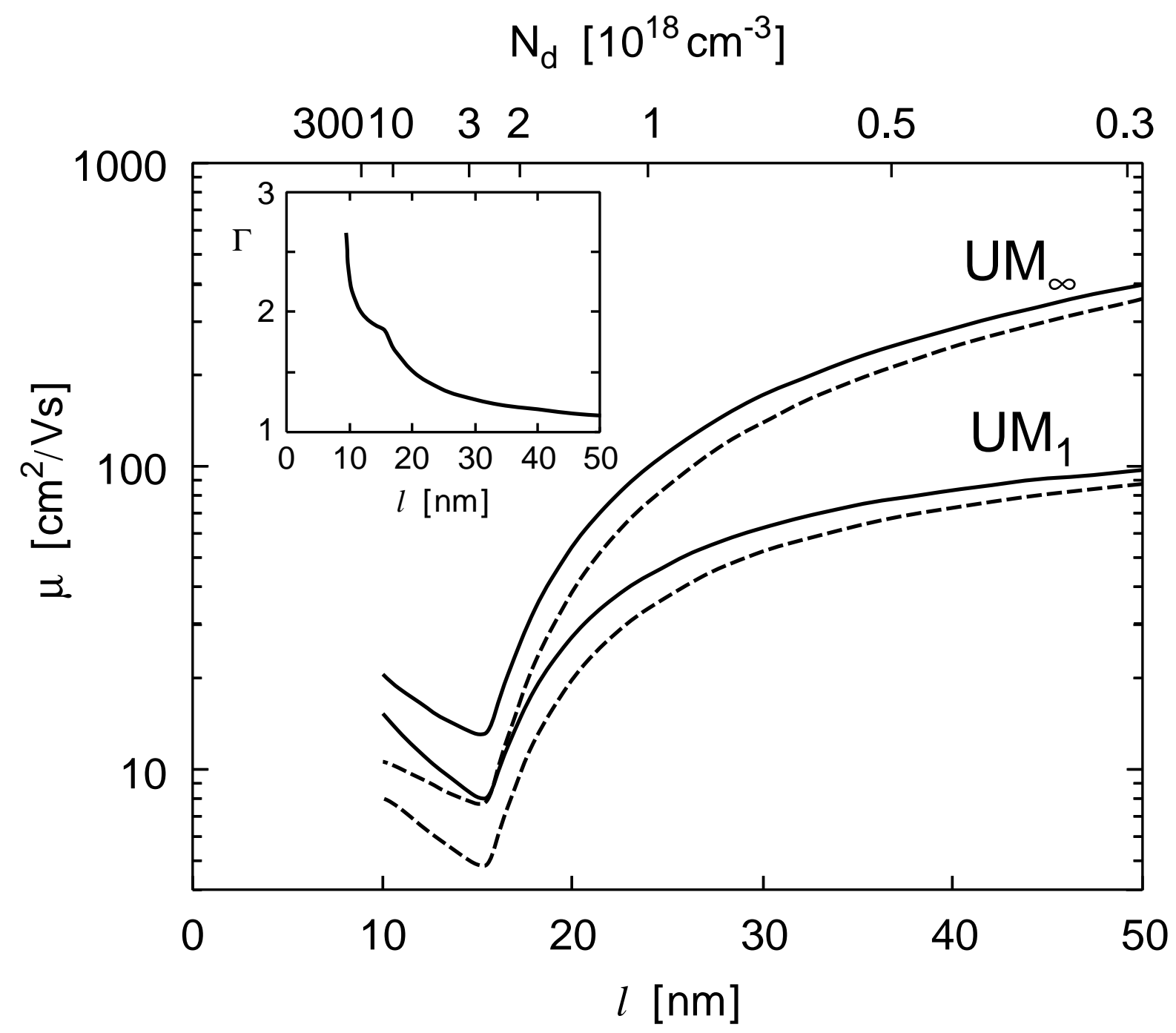

FIG. 3. Illustration of the effect of tunneling on the electron mobilities. Solid curves: unified-model mobilities of Fig. 2. Dashed curves: corresponding classical mobilities, calculated from Eq. (11) with $\Gamma=1$. Inset: $l$-dependence of the tunneling correction $\Gamma$. 


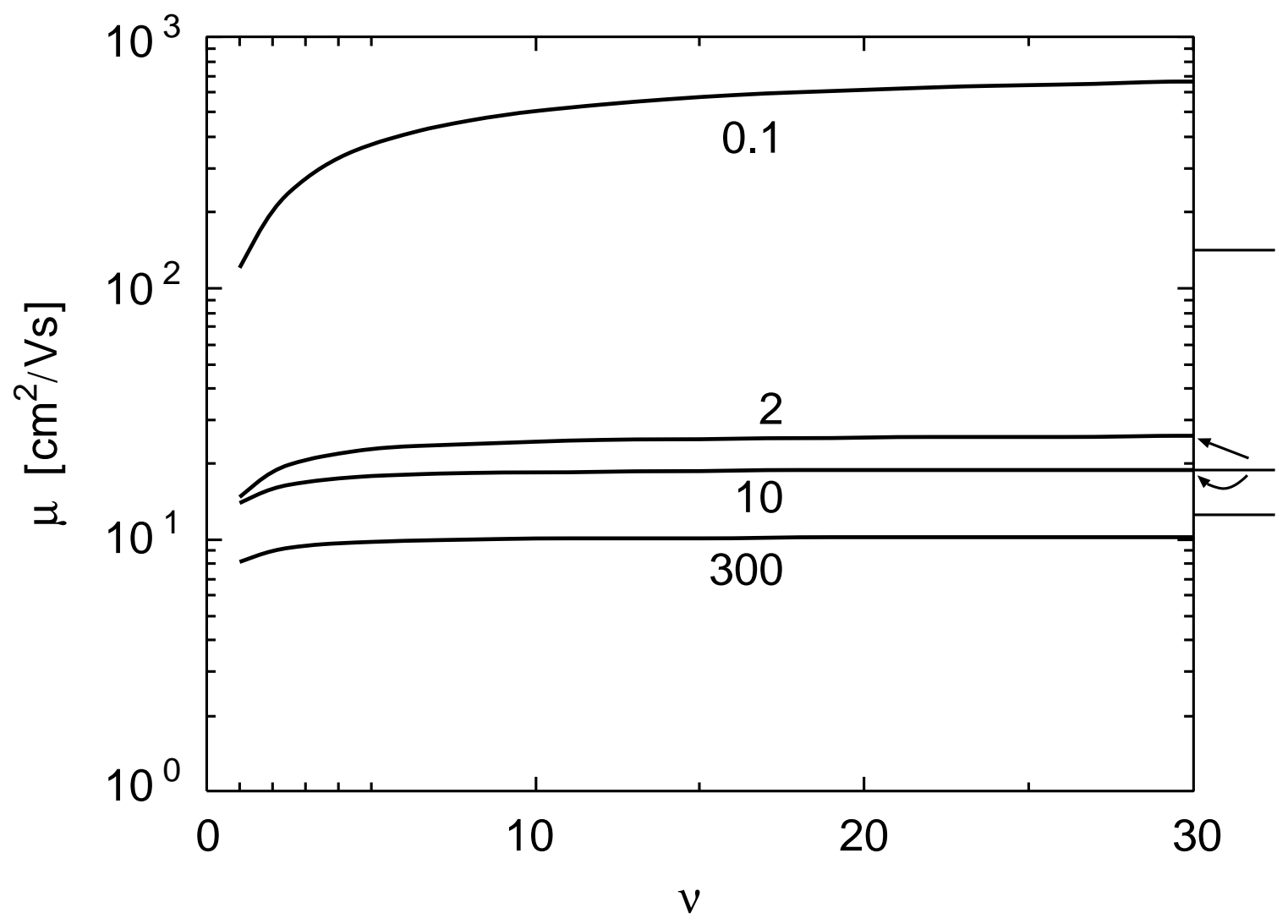

FIG. 4. Unified-model mobilities for n-type $\mu \mathrm{c}$-Si with grain length $s=15 \mathrm{~nm}$ at temperature $T=300 \mathrm{~K}$, calculated from Eq. (11) as a function of the number of grains, $\nu$, for different values of the donor concentration $N_{\mathrm{d}}$. The numbers attached to the curves are the $N_{\mathrm{d}}$-values in units of $10^{18} \mathrm{~cm}^{-3}$. For comparison, the corresponding mobilities in the thermionic-field-emission model, with $N_{\mathrm{d}}$ increasing from top to bottom, are indicated by bars at the right-hand ordinate. For the parameter values in the trapping-state density (13), see text. 


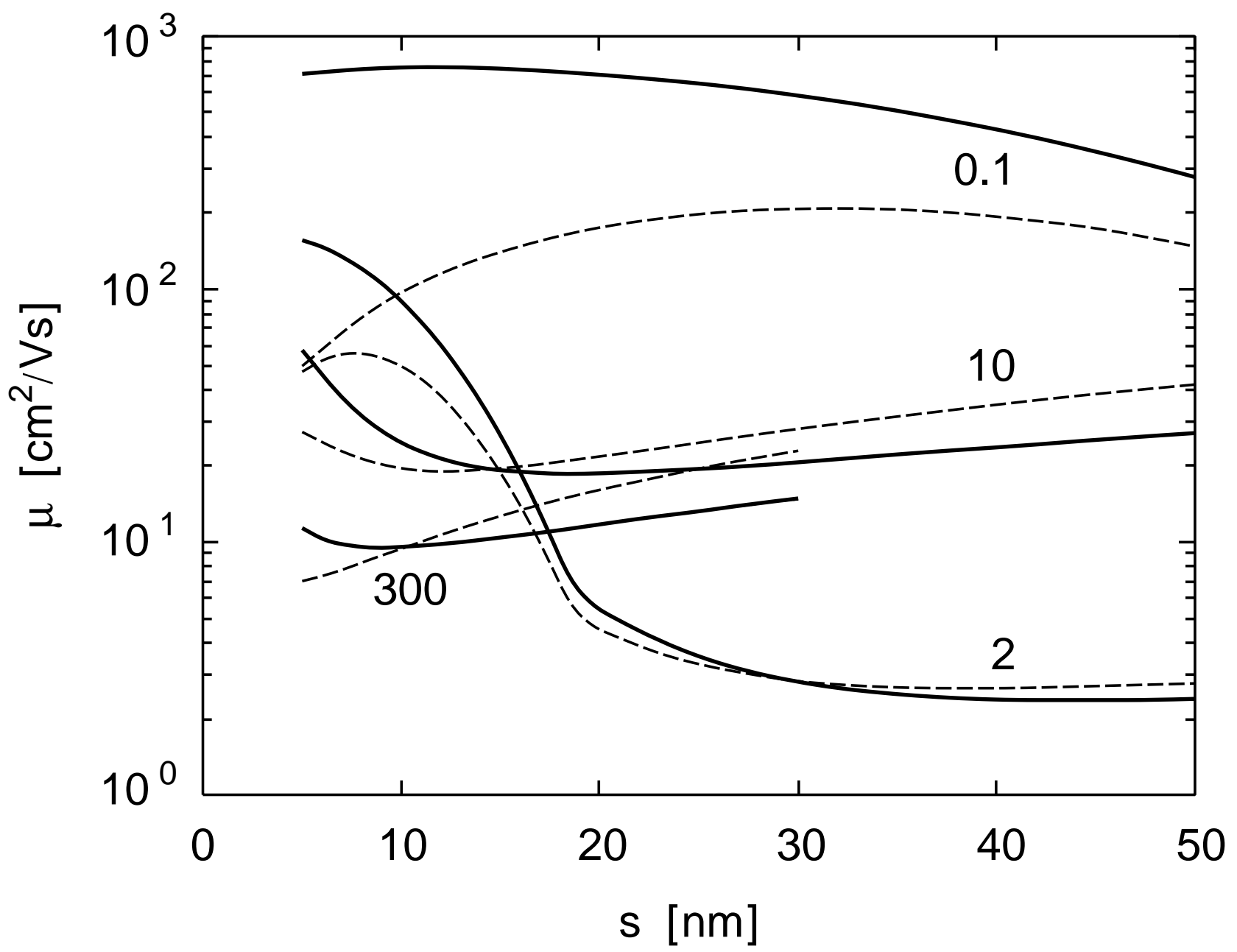

FIG. 5. Theoretical electron mobilities for n-type $\mu \mathrm{c}$-Si at temperature $T=300 \mathrm{~K}$ and different donor concentrations $N_{\mathrm{d}}$, plotted as a function of grain length $s$. Solid curves: mobilities calculated from the unified-model expression (11) for $\nu \rightarrow \infty$. Dashed curves: mobilities in the thermionic-field-emission model. The numbers attached to pairs of curves are the $N_{\mathrm{d}}$-values in units of $10^{18} \mathrm{~cm}^{-3}$. For the parameter values in the trapping-state density (13), see text. 


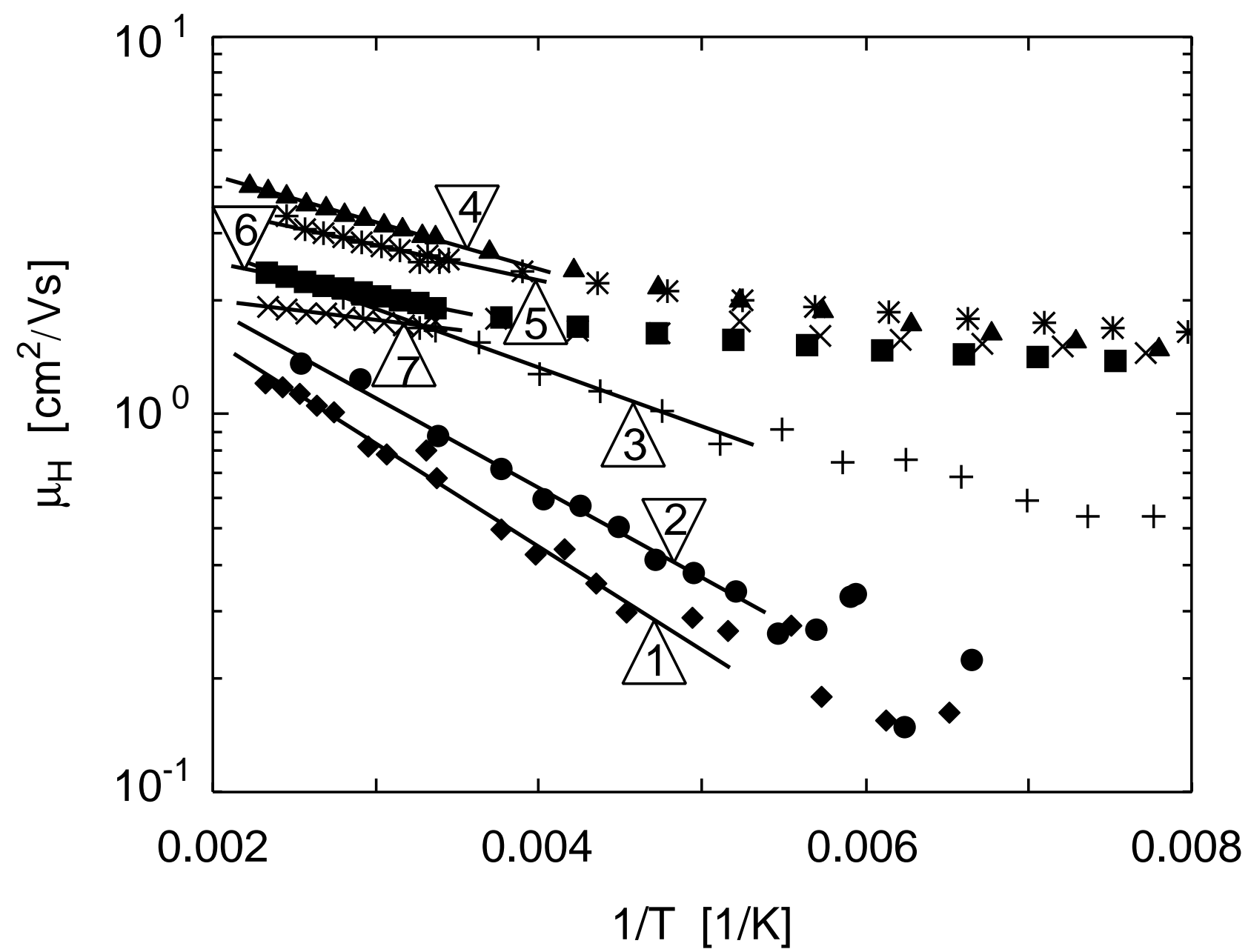

FIG. 6. Hall mobilities as a function of inverse temperature for thin films of phosphorus-doped $\mu \mathrm{c}-\mathrm{Si}: \mathrm{H}$ 19,20]. The straight lines fitted to the data in the high- $T$ range determine the preexponentials $\mu_{\mathrm{H}}^{0}$ and the activation energies $E_{\mathrm{a}}$ introduced in Eq. (14). The labels attached to the different lines correspond to the numbering of the samples in Table I. 


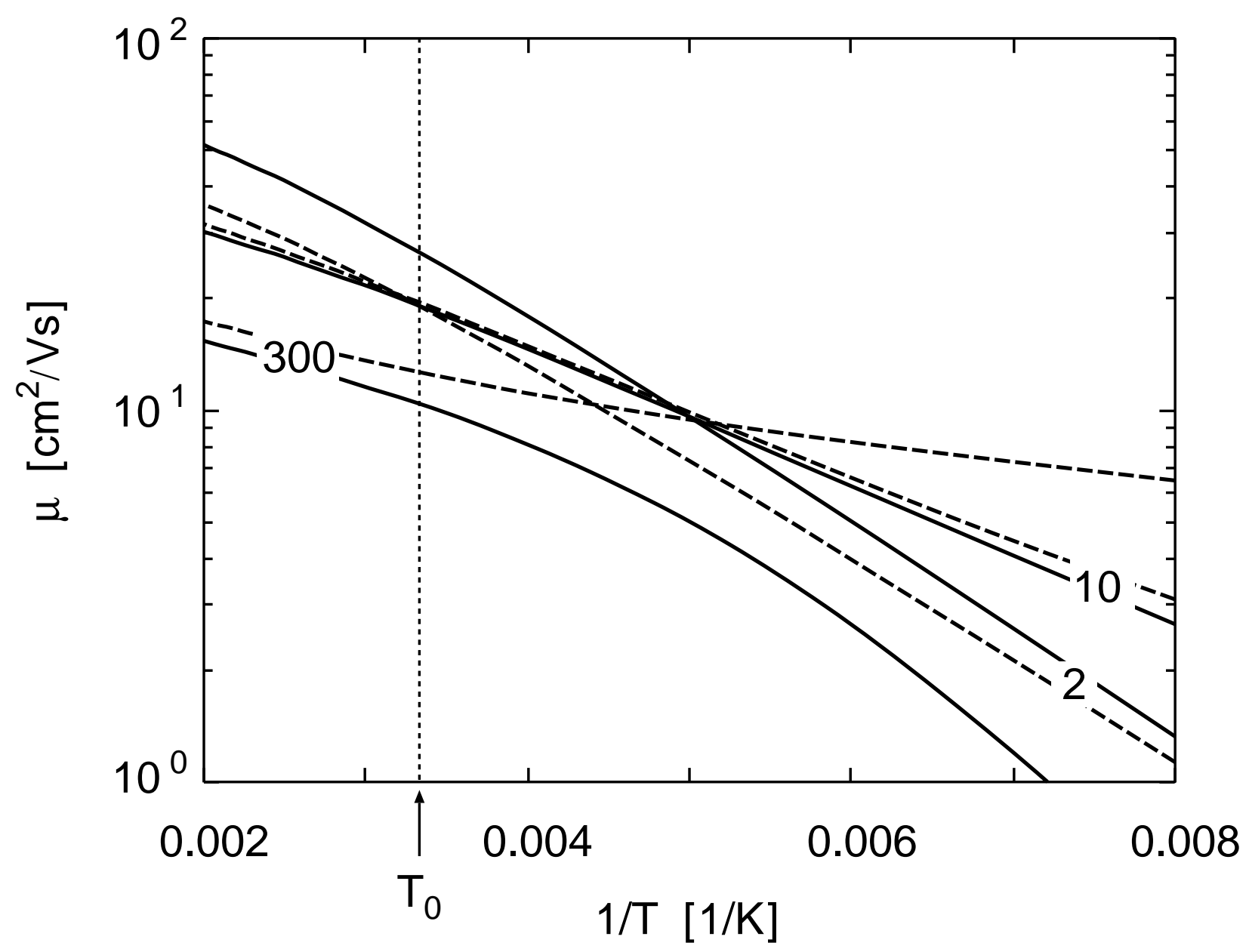

FIG. 7. Theoretical electron mobilities for n-type $\mu \mathrm{c}$-Si with grain length $s=15 \mathrm{~nm}$ and different donor concentrations $N_{\mathrm{d}}$, plotted as a function of inverse temperature, $1 / T$. Solid curves: mobilities calculated from the unified-model expression (11) for $\nu \rightarrow \infty$. Dashed curves: mobilities in the thermionic-field-emission model. The numbers attached to pairs of curves are the $N_{\mathrm{d}}$-values in units of $10^{18} \mathrm{~cm}^{-3}$. For the parameter values in the trapping-state density (13) and for the definition of $T_{0}$, see text. 


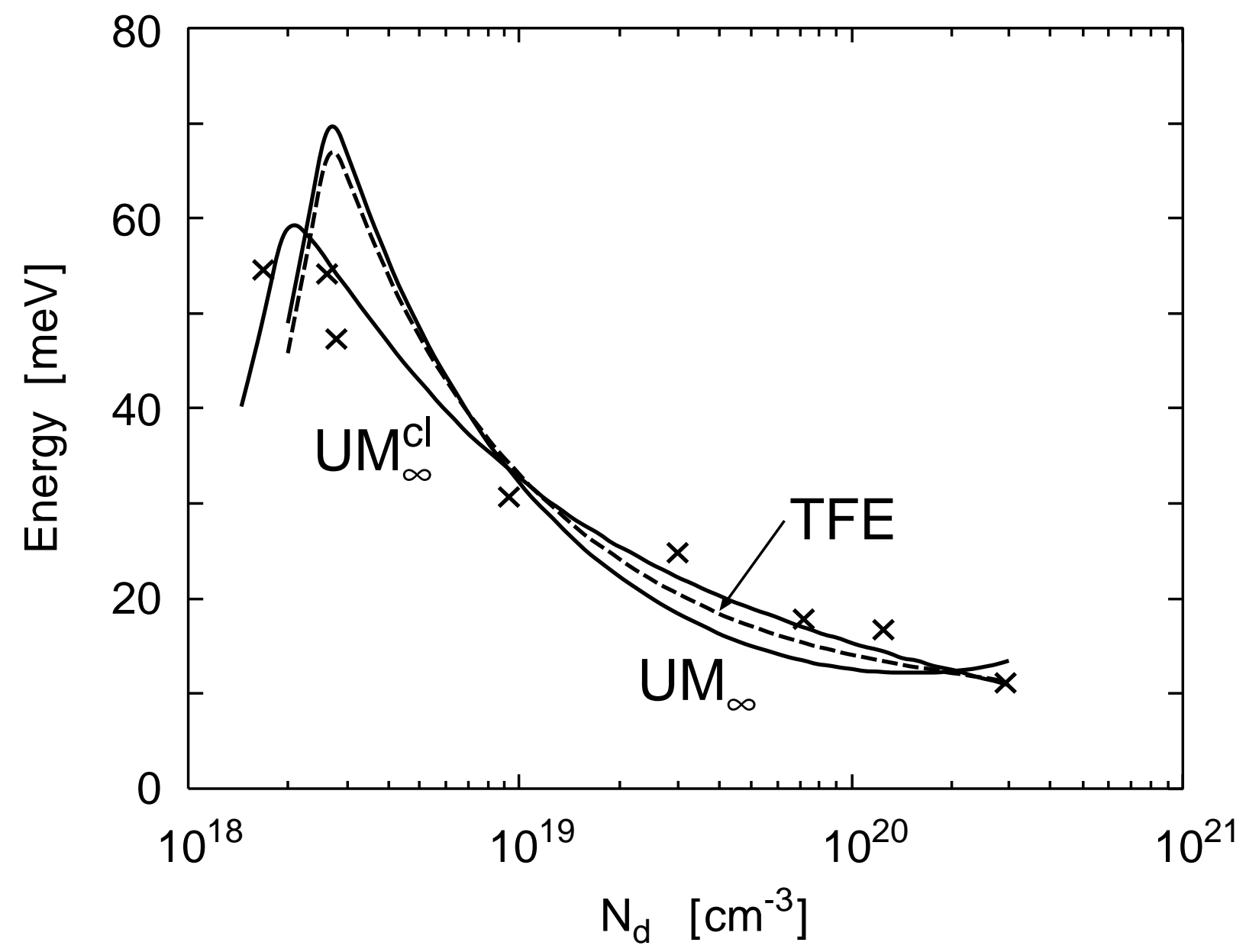

FIG. 8. Activation energies and effective barrier heights for n-type $\mu \mathrm{c}-\mathrm{Si}$ as a function of donor concentration $N_{\mathrm{d}}$. Symbols: activation energies $E_{\mathrm{a}}$ deduced from the Hall data (cf. Fig. 7 and Table I). Solid curves: effective barrier heights $E_{\mathrm{b}}^{\text {eff }}$ for $s=15 \mathrm{~nm}$, obtained by fitting expression (15) to the unified-model mobilities calculated from Eq. (11) at $T_{0}=300 \mathrm{~K}$; curve $\mathrm{UM}_{\infty}$ : result based on the full expression (11), curve $\mathrm{UM}_{\infty}^{\mathrm{cl}}$ : classical result $[\Gamma=1$ in Eq. (11)]. Dashed curve: effective barrier heights obtained from the thermionic-field-emission model. For the parameter values in the trapping-state density (13), see text. 


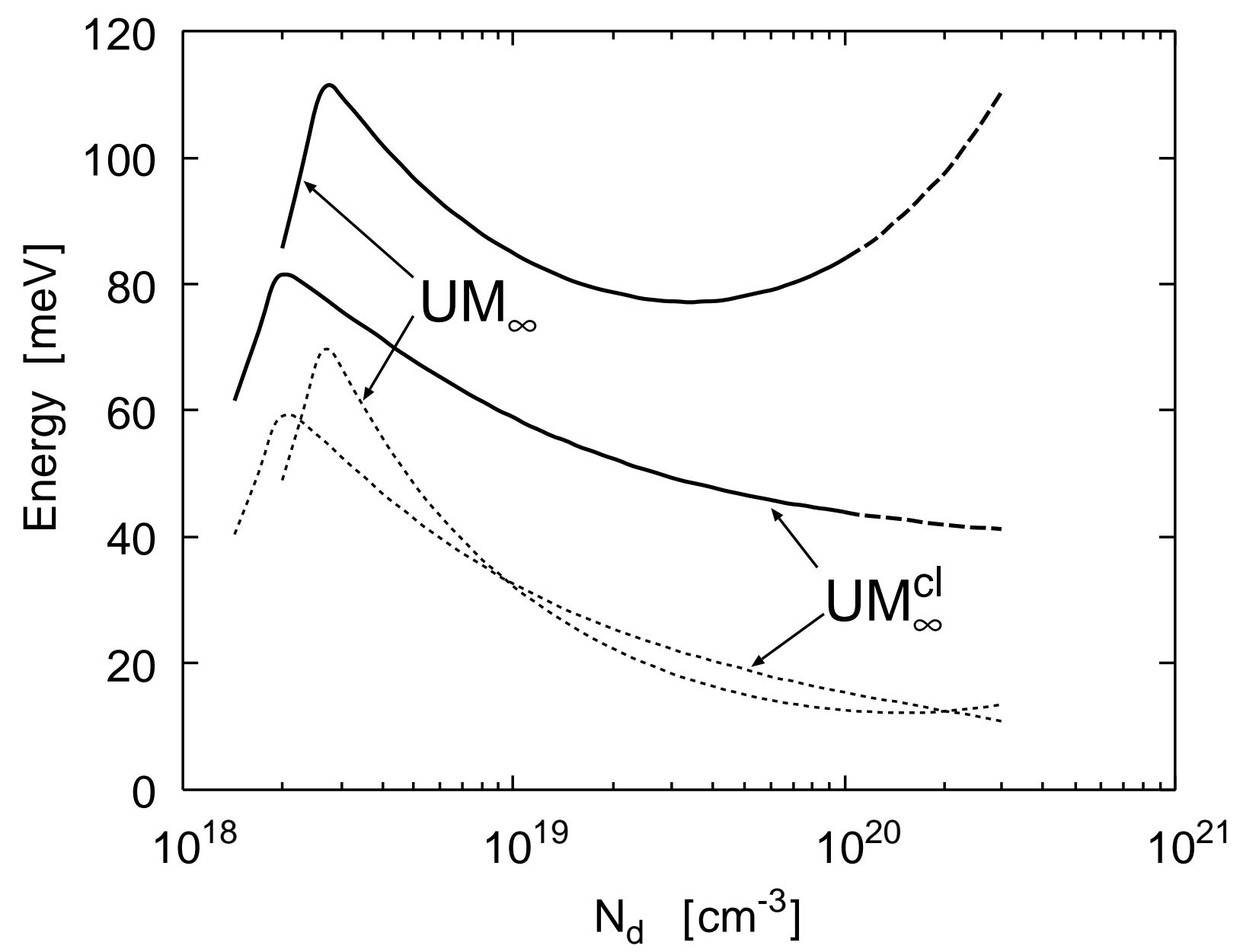

FIG. 9. Grain-boundary barrier heights for n-type $\mu \mathrm{c}-\mathrm{Si}$ as a function of donor concentration $N_{\mathrm{d}}$. Solid curves: true barrier heights $E_{\mathrm{b}}$ for the parameter values of Fig. 8, derived within the unified transport model; curve $\mathrm{UM}_{\infty}$ : result based on the full expression (11), curve $\mathrm{UM}_{\infty}^{\mathrm{cl}}$ : classical result $[\Gamma=1$ in Eq. (11)]; the meaning of the dashed parts of the curves is explained in the text. Dotted curves: corresponding effective barrier heights $E_{\mathrm{b}}^{\mathrm{eff}}$ (cf. Fig. 8). 